\title{
Constrictive epicarditis following Coxsackie virus infection
}

\author{
D. K. C. COOPER and M. F. S T URR I D G
}

The Middlesex Hospital, Londion W1

\begin{abstract}
Cooper, D. K. C. and Sturridge, M. F. (1976). Thorax, 31, 472-474. Constrictive epicarditis following Coxsackie virus infection. A case is presented of the rare condition of constrictive epicarditis, which was treated successfully by removal of the epicardium. This appears to be the first account of constrictive epicarditis following proven Coxsackie viral infection.
\end{abstract}

Constrictive pericarditis is a complication of viral pericarditis. The suggestion that it may follow Coxsackie infection came from Robertson and Arnold $(1962,1965)$, and the first proven case was described in 1965 by Gibbons, Goldbloom, and Dobell. Further cases since then were reviewed briefly by Matthews and his colleagues (1970). The clinical features of non-tuberculous constrictive pericarditis have been classified recently by Ikram and his colleagues (1974).

Constrictive epicarditis appears to have been described previously on only three occasions. It has not been reported previously in a patient following proven Coxsackie viral pericarditis.

\section{CASE REPORT}

A 33-year-old man was admitted to The Middlesex Hospital in September 1972 as an emergency with a history of intermittent dull central chest pain exacerbated by movement and by respiration. The pain had been troublesome for only a few days and was associated with low-grade pyrexia. He gave no history of rheumatic fever or tuberculosis. The patient's wife had been unwell the previous week with malaise and myalgia but had recovered without medical attention.

On examination the patient looked well and was not distressed. Apart from an apical fourth heart sound there were no abnormal physical signs, and the electrocardiogram showing a prolonged P-R interval and minimal ST elevation in standard lead II and V3 was the only abnormal routine investigation. A chloroform resistant virus, identified as Coxsackie B5, was isolated by tissue culture from both stool and throat swabs. The chest pain and pyrexia settled within 48 hours without treat- ment, and the patient was discharged free of $\frac{d}{\omega}$ symptoms four days later.

Three months later the patient developed an $\vec{\oplus}$ influenza-like illness with tiredness and ankle. swelling. He was readmitted and on examination was found to be in atrial fibrillation with a pulse rate of $100 /$ minute. His blood pressure was $130 / 80 \mathrm{mmHg}$ with no paradox. The venous pressure was raised, the liver was palpable to the $\stackrel{\mathbb{Q}}{\stackrel{D}{2}}$ umbilicus, and there was slight pitting oedema of $\overrightarrow{\vec{A}}$ the ankles; other clinical findings were normal. 3 Blood tests revealed haemoglobulin $14.2 \mathrm{~g} / \mathrm{dl}$, white cell count $6500 / \mathrm{mm}^{3}(76 \%$ polymorphs, $17 \%$ lymphocytes, and $7 \%$ monocytes), and ESR $12 \mathrm{~mm}$ in one hour. A radiograph showed ao cardiothoracic ratio of 0.51 with no pericardial $\left.\right|_{x} ^{0}$ calcification. Electrocardiography confirmedoatrial fibrillation and showed that a voltage re 3 . duction had occurred since the previous admission? 16 months earlier. Echocardiography showed 3 evidence of anterior and posterior pericardialo effusions.

Right heart catheterization and angiography气 were performed, revealing raised right-sided pressures (right atrium $28 \mathrm{mmHg}$, right ventricle $50 / 10 \mathrm{mmHg}$, and pulmonary artery $50 / 30 \mathrm{mmHg}$ ). Cine-angiography suggested a large pericardial effusion and excluded any atrial fill-o ing defects. Left ventricular contraction appearedo normal, though end-systolic and diastolic volumes were small. After the aspiration of $250 \mathrm{ml}$ of bloodstained fluid from the pericardial space the 0 right-sided pressures showed little change ando remained raised; cardiac pulsations, however, be $-\mathbb{D}$ came more evident. The fluid obtained contained迟 a few mesothelial cells and lymphocytes. $\mathrm{A}_{\bar{\sigma}}^{\circ}$ 
diagnosis of constrictive pericarditis was made. In view of the previous history and the absence of calcification on chest radiography, the pericarditis was considered to be viral in origin. The patient was given digitalis and treated with diuretics preoperatively.

On 31 January 1974 a small left anterior thoracotomy was performed via the lower border of the fifth rib. There was a moderate left pleural effusion. The pericardium was thickened to 1.5$2 \mathrm{~mm}$ and inflamed but was loose around the heart. There were no adhesions between the pericardium and the epicardium. The pericardial sac contained some $250 \mathrm{ml}$ of straw-coloured fluid, and the heart was small and covered with a thin, pale fibrinous layer, as one might expect with such an effusion. There was no evidence of constriction of the heart by the pericardium as usually defined and therefore no indication for pericardiectomy. At this stage the condition was attributed to reduced compliance of the ventricles and biopsies were sought in the hope of finding an explanation.

A biopsy was taken from the parietal pericardium, and a very small myocardial biopsy was taken from the apex of the left ventricle for histological examination. After this had been done it was noticed that the myocardium bulged through a hole in the epicardium, suggesting the possibility that the epicardium was constric'ing the heart. The epicardium was therefore progressively removed from the entire surface of the ventricles and the posterior aspect of the left atrium. The right atrium was cleared as far as the roots of the venae cavae. Following this, as dissection proceeded, the heart was noted to become progressively larger in volume with increased diastolic filling. The parietal pericardium was not resected and the chest wound was closed in the usual manner.

On the first postoperative day the patient developed supraventricular tachycardia which responded to digitalis, but otherwise his progress was uneventful. He was discharged on the 22 nd postoperative day in controlled atrial fibrillation. The jugular venous pressure was not raised but the liver was still palpable. Residual cardiomegaly noted in the chest film suggested a cardiomyopathy from the same viral cause as the epicarditis.

Studies on the excised epicardium for viruses were negative. The pericardial biopsies showed fibrous thickening with a moderate degree of vascularity; foci of acute and chronic inflammatory cells were present but there were no specific features.
The patient was readmitted four months later and DC cardioversion was successfully performed. There were no abnormal physical findings on this admission. Subsequent ECGs showed sinus rhythm with first-degree heart block, the P-R interval being $240 \mathrm{~ms}$.

He was last seen in March 1975 when he was asymptomatic and had no abnormal physical signs, the jugular venous pressure being normal.

\section{DISCUSSION}

Constrictive epicarditis is extremely rare. It was originally described by Burchell in 1954, though it was not discovered until necropsy. Both the parietal pericardium and the epicardium were thickened but there were no adhesions between the two; the pericardium was distended under high pressure with a bloody effusion, but it was thought that the epicardium was constrictive.

Edwards (1961) gave further details of Burchell's case and reported a similar case which also was not diagnosed until necropsy. The patient was an adult with a pericardial effusion, but after aspiration the venous pressure remained high. A partial pericardiectomy was therefore performed but the patient failed to recover from operation. At necropsy there was evidence of chronic fibrous pericarditis without adhesion between the two layers. It was suggested that this patient's disease started as non-specific pericarditis which, as a result of organization of exudate, led to two phenomena-dense fibrous replacement of the exudate over the epicardium and haemorrhage from the organizing exudate.

In both of the above cases the cause of the pericardial disease was attributed to idiopathic acute pericarditis', but in retrospect it is possible that it was of viral origin.

The only case of successfully treated constrictive epicarditis was that of a 10-month-old infant reported by Castellanos et al. (1969). The disease started as acute staphylococcal osteomyelitis of the left humerus which was drained. Posioperatively, bilateral pleural effusions and a purulent pericarditis developed. In spite of vigorous antibiotic therapy, constrictive pericarditis ensued and pericardiectomy was performed. At operation both the pericardium and the epicardium were thickened but the two layers were not significantly adherent. The heart was easily freed, and immediately the cardiac action was seen to be more vigorous. The result of surgical intervention was clinically dramatic.

We feel that constriction following viral pericarditis is more difficult to diagnose than that 
which follows tuberculous pericarditis and which is often associated with extensive pericardial calcification. In viral pericarditis, critical constriction can develop quite rapidly. The heart shadow may be radiographically enlarged and substantial amounts of fluid may have been aspirated sufficiently recently to make the diagnosis of cons'rictive pericarditis seem untenable.

Echocardiography reduces the chance of effusions that can be treated successfully by aspiration alone being overlooked, but this case and that of Burchell illustrate that this procedure does not relieve patients with constrictive epicarditis. An awareness of the possibility of this condition should prevent such cases being overlooked. A comparison of catheter data before and after pericardiocentesis should always be undertaken in patients whose 'medical' management seems unsatisfactory. Surgical exploration is advised whenever a more satisfactory haemodynamic state is not achieved, and at operation, the release of pericardial fluid and the absence of pericardial adhesions should not exclude critical examination of the epicardium.

\section{REFERENCES}

Burchell, H. B. (1954). Problems in the recognition and treatment of pericarditis. Journal-Lancet, 74, 465 .
Castellanos, A., Mercado, H., Traggis, D. G., and Altman, D. H. (1969). Successful surgical treat-? ment of constrictive epicarditis in a ten-monthold infant: a case report. Acta Cardiologica, 24, 음 633.

Edwards, J. E. (1961). An Atlas of Acquired Diseases of the Heart and Great Vessels, Vol. 1, pp. 436441. Saunders, Philadelphia and London.

Gibbons, J. E., Goldbloom, R. B., and Dobell, A. R. C. (1965). Rapidly developing pericardialconstriction in childhood following acute non- $\vec{\omega}$ specific pericarditis. American Journal of Car-ळ diology, 15, 863.

Ikram, H., Banim, S. O., and Mackey, A. R. (1974). Clinical features of non-tuberculous constrictive.pericarditis. Thorax, 29, 204.

Matthews, J. D., Cameron, S. J., and George, M. (1970). Constrictive pericarditis following Coxsackie virus infection. Thorax, 25, 624.

Robertson, R. and Arnold, C. R. (1962). Constrictivepericarditis with particular reference to etiology. Circulation, 26, 525. and - (1965). Acute constrictive pericarditis. $\stackrel{\text { S }}{\rightarrow}$ Journal of Thoracic and Cardiovascular Surgery, 49, 91.

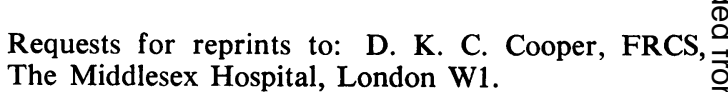

